\title{
High-Spin States Populated in Deep-Inelastic Reactions
}

\author{
S. Mohammadi ${ }^{1,2}$, Zs. Podolyák ${ }^{1}$, G. de Angelis ${ }^{3}$, M. Axiotis ${ }^{3}$, D. Bazzacco ${ }^{4}$, \\ P.G. Bizzeti ${ }^{5}$, F. Brandolini ${ }^{4}$, R. Broda ${ }^{6}$, D. Bucurescu ${ }^{7}$, E. Farnea ${ }^{3}$, W. Gelletly ${ }^{8}$, A. Gadea ${ }^{3}$, \\ M. Ionescu-Bujor ${ }^{7}$, A. Iordachescu ${ }^{7}$, Th. Kröll ${ }^{3}$, S. Longdown ${ }^{8}$, S. Lunardi ${ }^{4}$, N. Marginean ${ }^{3}$, \\ T. Martinez ${ }^{3}$, N.H. Medina ${ }^{9}$, B. Quintana ${ }^{10}$, P.H. Regan ${ }^{8}$, B. Rubio ${ }^{11}$, C.A. Ur ${ }^{4}$, \\ J.-J. Valiente Dobón ${ }^{8}$, P.M. Walker ${ }^{8}$, and Y.H. Zhang ${ }^{3}$ \\ ${ }^{1}$ Department of Physics, University of Surrey, Guildford, GU2 7XH, UK \\ ${ }^{2}$ Department of Physics, University of Payam-Noor, Fariman 93914, Iran \\ ${ }^{3}$ Laboratori Nazionali di Legnaro, INFN, Legnaro, Italy \\ ${ }^{4}$ Dipartimento di Fisica and INFN, Padova, Italy \\ ${ }^{5}$ Dipartimento di Fisica and INFN, Firenze, Italy \\ ${ }^{6}$ Niewodniczanski Institute of Nuclear Physics, Krakow, Poland \\ ${ }^{7}$ Insitute of Physics and Nuclear Engineering, Bucharest, Romania \\ ${ }^{8}$ Department of Physics, University of Surrey, Guildford GU2 7XH, UK \\ ${ }^{9}$ Laboratório Pelletron-IFUSP, São Paulo, SP, Brazil \\ ${ }^{10}$ University of Salamanca, Spain \\ ${ }^{11}$ Instituto di Fisica Corpuscular, Valencia, Spain
}

Received on 12 September, 2003

\begin{abstract}
High spin states in the neutron rich ${ }^{188}$ Os and ${ }^{190}$ Os nuclei have been populated using the ${ }^{82} \mathrm{Se}+{ }^{192}$ Os deepinelastic reaction. The level schemes are extended up to spin $I \approx 21$. The observed new structures are tentatively interpreted as fragments of rotational bands built on multi-quasiparticle configurations.
\end{abstract}

\section{Introduction}

Fusion-evaporation reactions provide the standard mechanism to populate states with high angular momentum. However, by using stable beam-target combinations beta-stable and neutron-rich nuclei cannot be studied. Neutron-rich nuclei with mass $\mathrm{A}<150$ can be studied in spontaneous and induced fission. Projectile fragmentation has proven to be an efficient method of populating nuclei far from the valley of stability. However, in the case of heavy nuclei this method is still limited to species with isomeric states, needed to enhance the sensitvity of the technique (see for example [1]). Deep-inelastic reactions are the most general reaction mechanism which can be used to study neutron-rich nuclei and are able to populate relatively high-spin states.

We used the ${ }^{82} \mathrm{Se}+{ }^{192} \mathrm{Os}$ binary reaction to study nuclei both in the vicinity of the ${ }^{82} \mathrm{Se}$ beam and ${ }^{192}$ Os target. The study of nuclei around the $\mathrm{N}=50$ shell closure has given new experimental indications about the persistence of this shell gap down to $\mathrm{Z}=32$ [2]. The structure of nuclei in the $A \approx 180-190$ region is characterized by the presence of high-angular momentum intrinsic, i.e. noncollective, states. Such states compete with the collective excitations because the proton and neutron Fermi levels are among orbitals with large angular-momentum projections $(\Omega)$ on the symmetry axis, allowing states with large values of $K=\Sigma \Omega$ to be formed [3]. As part of a systematic study of the mass $A \approx 180-190$ nuclei, some new results on the stable ${ }^{188,190}$ Os nuclei, obtained by using binary reactions, are presented in this paper.

\section{Experiment}

Excited states in the vicinity of both the ${ }^{82} \mathrm{Se}$ beam and the ${ }^{192}$ Os target nuclei have been populated using heavyion multi-nucleon transfer reactions and studied through $\gamma$ ray spectroscopy in a "thick target" [4] measurement. The combination of the Tandem-XTU and the superconducting LINAC ALPI accelerators at the Laboratori Nazionali di Legnaro, Italy, was used to accelerate a beam of ${ }^{82} \mathrm{Se}$ ions to an energy of $460 \mathrm{MeV}$. The ${ }^{192} \mathrm{Os}$ target, isotopically enriched to $99.0 \%$, had a thickness of $50 \mathrm{mg} / \mathrm{cm}^{2}$ on a $0.2 \mathrm{~mm}$ Ta backing, which is sufficient to stop all reaction fragments. High fold $\gamma-\gamma$ coincidences were acquired with the $4 \pi$ spectrometer GASP [5] consisting of 40 Compton-suppressed, large-volume germanium detectors with an inner BGO ball 


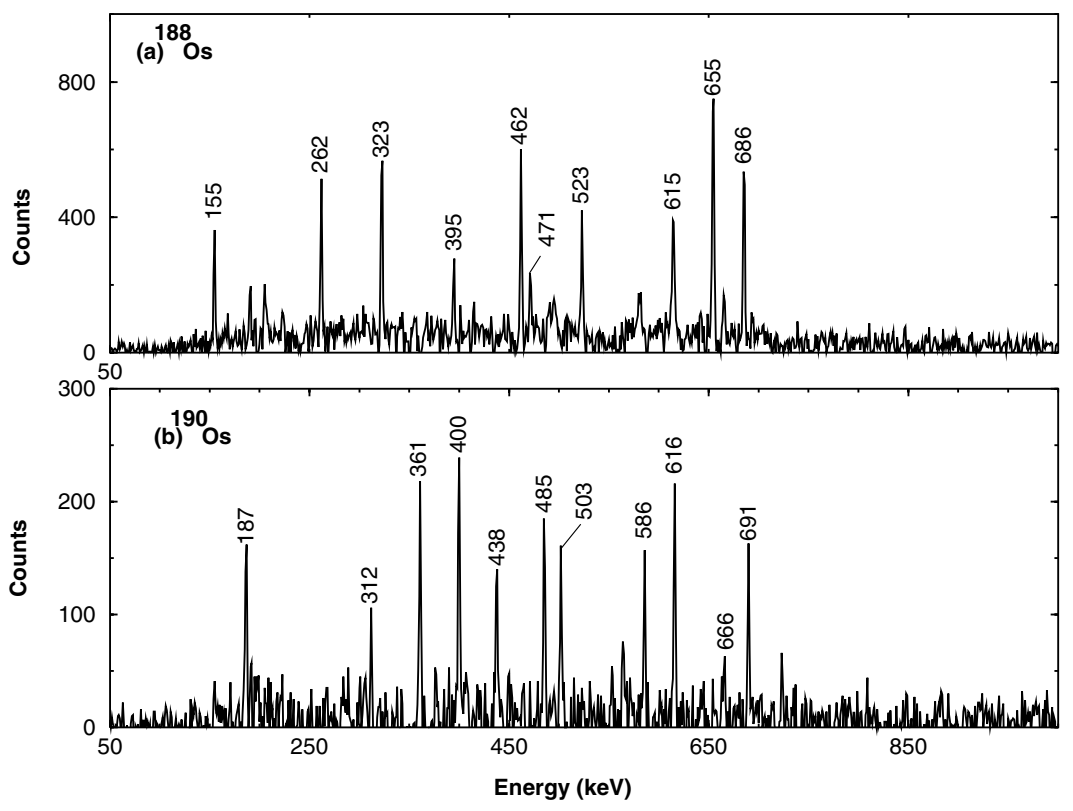

Figure 1. Double gated coincidence spectra for ${ }^{188,190}$ Os. (a) In ${ }^{188}$ Os the gates are on the $(155,323,462 \mathrm{keV}) \& 574 \mathrm{keV}$ transitions. (b) In ${ }^{190}$ Os the gates are on the $(187,361,503 \mathrm{keV}) \& 589 \mathrm{keV}$ transitions.

acting as a multiplicity filter and a total-energy spectrometer. Events were collected on tape during five days of beam time under the conditions that a minimum of three Comptonsuppressed Ge detectors and two BGO elements from the multiplicity filter fired in coincidence. With a beam current of 1 particle-nA, the event rate was $\approx 4 \mathrm{kHz}$ and the singles rate in the germanium detectors $\approx 12 \mathrm{kHz}$. With such a setup $\gamma$-rays from the deexciting target-like and projectilelike fragments were detected. After gain matching for all the detectors, the coincidence data were sorted into fully symmetrized matrices and cubes for off-line analysis. Since all recoiling fragments were stopped in the target, Doppler broadening prevented the observation of transitions deexciting short-lived states and only the $\gamma$ decay with lifetimes longer than the slowing-down time of the recoiling nuclei $(\approx 1 \mathrm{ps})$ could be studied. Thus, no Doppler correction was necessary when sorting the data.

\section{Results}

Two- and three-dimensional $\gamma$ matrices were used to construct the level schemes of ${ }^{188,190}$ Os. Typical coincidence spectra are shown in Fig. 1. Partial level schemes of ${ }^{188}$ Os and ${ }^{190} \mathrm{Os}$, together with the lighter ${ }^{184,186}$ Os isotopes [6, 7], are presented in Fig. 2.

In ${ }^{188}$ Os the levels up to spin $10^{+}$of the ground state band are well established $[8,9,10]$ and were confirmed in the present experiment. C.Y. Wu et al. [9] reported two transitions, with energies of $699 \mathrm{keV}$ and $760 \mathrm{keV}$, populating the yrast $10^{+}$state in Coulomb excitation. We did not observe these transitions. Instead, we confirm the existence of the $686 \mathrm{keV}$ transition, previously reported by R.A. Warner et al. [11]. The levels above the $12^{+}$state are seen in this experiment for the first time. The properties of the gamma ray transitions belonging to ${ }^{188} \mathrm{Os}$ are summarized in Table I.

TABLE I: $\gamma$-ray transition energies, relative intensities and level assignments in ${ }^{188}$ Os.

\begin{tabular}{cccc}
\hline$E_{\gamma}(\mathrm{keV})$ & $I_{\gamma}(\mathrm{rel})$. & $I_{i}^{\pi a)}$ & $I_{f}^{\pi a}$ \\
\hline 655 & $1000(80)$ & $10^{+}$ & $8^{+}$ \\
686 & $710(48)$ & $12^{+}$ & $10^{+}$ \\
615 & $531(45)$ & $14^{+}$ & $12^{+}$ \\
523 & $384(38)$ & $16^{+}$ & $14^{+}$ \\
262 & $274(26)$ & $18^{+}$ & $16^{+}$ \\
395 & $243(40)$ & $19^{+}$ & $18^{+}$ \\
471 & $240(44)$ & $20^{+}$ & $19^{+}$ \\
495 & $182(64)$ & $21^{+}$ & $20^{+}$ \\
\hline
\end{tabular}

a) The spin-parity values are tentative. They are based on comparison with the lighter osmium isotopes. 


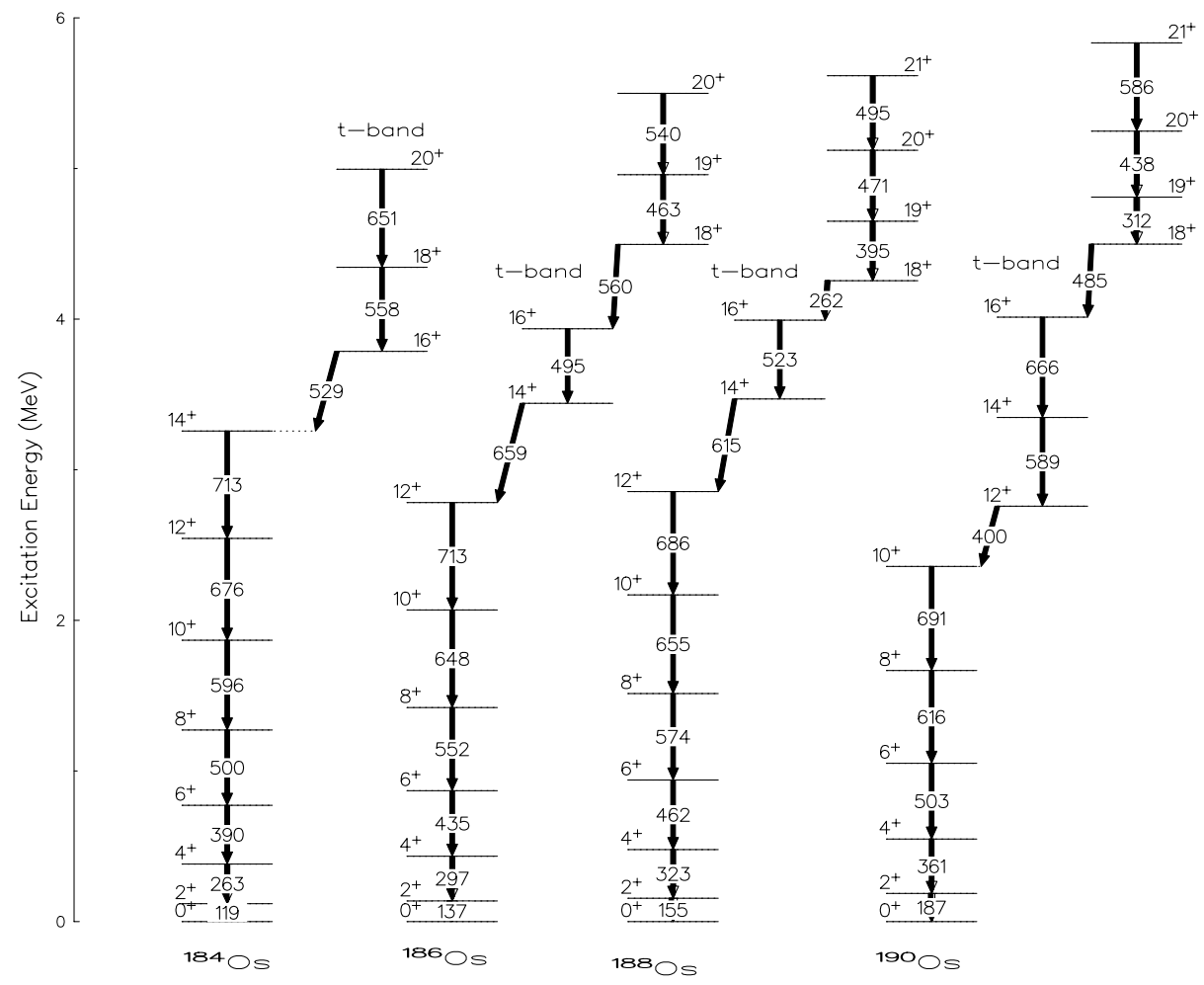

Figure 2. Positive parity yrast structures of ${ }^{184,186,188,190}$ Os nuclei. The high spin states in ${ }^{188,190}$ Os are from the present work. The spinparity and configuration assignmenst are tentative and based on comparison with ${ }^{184,186}$ Os [6,7]. For alternative configuration assignments see the text.

In ${ }^{190}$ Os the levels up to spin $10^{+}$of the ground state band are known from earlier works $[9,10,12]$. Here again we did not see the $769 \mathrm{keV}$ and $654 \mathrm{keV} \gamma$-ray transitions populating the $10^{+}$state, as proposed by C.Y. Wu et al. [9]. Levels above the $10^{+}$state are seen for the first time in the present work. The properties of the gamma ray transitions belonging to ${ }^{190} \mathrm{Os}$ are summarized in Table II.

TABLE II. $\gamma$-ray transition energies, relative intensities and level assignments in ${ }^{188} \mathrm{Os}$.

\begin{tabular}{cccc}
\hline$E_{\gamma}(\mathrm{keV})$ & $I_{\gamma}(\mathrm{rel})$. & $\left.I_{i}^{\pi a}\right)$ & $\left.I_{f}^{\pi a} a\right)$ \\
\hline 400 & $100(12)$ & $12^{+}$ & $10^{+}$ \\
589 & $96(10)$ & $14^{+}$ & $12^{+}$ \\
666 & $96(7)$ & $16^{+}$ & $14^{+}$ \\
485 & $88(12)$ & $18^{+}$ & $16^{+}$ \\
312 & $45(9)$ & $19^{+}$ & $18^{+}$ \\
438 & $64(10)$ & $20^{+}$ & $19^{+}$ \\
586 & $59(8)$ & $21^{+}$ & $20^{+}$ \\
\hline
\end{tabular}

a) The spin-parity values are tentative. They are based on comparison with the lighter osmium isotopes.

\section{Discussion}

As first step of the configuration assignment we compare the new structures observed in ${ }^{188,190}$ Os with the lighter isotopes (see fig.2), where more experimental data are available. In both ${ }^{184,186}$ Os the groundstate band is crossed by a so-called t-band, based on the two-neutron $\nu 11 / 2^{+}[615] 9 / 2^{+}[624]$ configuration. The assignments were based on experimentally determined branching ratios, lifetimes of the $K^{\pi}=10^{+}$bandheads, and comparison with blocked BCS calculations [6, 7]. The yrast $18^{+}$state in ${ }^{186} \mathrm{Os}$ was interpreted as a $\mathrm{K}^{\pi}=18^{+}$four neutron $\nu 11 / 2^{+}[615] 9 / 2^{+}[624] 9 / 2^{-}[505] 7 / 2^{-}[503]$ state. Based on the similarities between the yrast structures of the osmium isotopes, we propose that the $14^{+}$and $16^{+}$ states in ${ }^{188} \mathrm{Os}$, and $12^{+}, 14^{+}, 16^{+}$states in ${ }^{190} \mathrm{Os}$ are members of the two-neutron $\nu 11 / 2^{+}[615] 9 / 2^{+}[624]$ band. In the same way the states with spin-parity $18^{+}, 19^{+}, 20^{+}, 21^{+}$may have the four neutron $\nu 11 / 2^{+}[615] 9 / 2^{+}[624] 9 / 2^{-}[505] 7 / 2^{-}$[503] configuration.

In order to verify whether the assignment based on systematics is reasonable, multi-quasiparticle calculations [13] have been performed using blocked BCS pairing without residual interaction. According to the calculations the $\mathrm{K}^{\pi}=9^{-} \nu 11 / 2^{+}[615] 7 / 2^{-}[503]$ intrinsic state is $\approx 800 \mathrm{keV}$ below the $\mathrm{K}^{\pi}=10^{+} \nu 11 / 2^{+}[615] 9 / 2^{+}[624]$ state, both in 
${ }^{188}$ Os and ${ }^{190}$ Os nuclei. However, the lack of observation of low energy dipole transitions favours the $\mathrm{K}^{\pi}=10^{+}$assignment. Furthermore, the $9^{-}$bandhead decay is expected to be isomeric, and may be too long lived to be seen in the present analysis,

We couldn't establish the level scheme of ${ }^{192}$ Os up to high spins, although its excited states must have been populated with high cross sections. This might be explained by the existence of a $\mathrm{K}^{\pi}=10^{-} T_{1 / 2}=5.9 \mathrm{~s}$ yrast isomer at $2015 \mathrm{keV}$ excitation energy, interpreted as having the $\nu 11 / 2^{+}[615] 9 / 2^{-}[505]$ configuration. However, a similar yrast isomer with $T_{1 / 2}=9.9 \mathrm{~min}$ at $1705 \mathrm{keV}$ exists also in ${ }^{190}$ Os [14]. It seems that in ${ }^{192}$ Os the high spin states decay via the long-lived isomer, whereas in ${ }^{190}$ Os the situation is different.

The data analysis is still in progress. Therefore, new experimental results (for example on angular distributions) and theoretical considerations might provide a more complete interpretation.

\section{Acknowledgments}

The authors thank the staff of the ALPI linear accelerator, Legnaro, for providing a high quality beam. Zs.P. acknowledges the receipt of an EPSRC Advanced Fellowship Award (GR/A10789/01).

\section{References}

[1] Zs. Podolyák et al., Phys. Lett. B491, 225 (2000).

[2] Y.H. Zhang et al., to be published.

[3] P.M. Walker and G.D. Dracoulis, Nature (London) 399, 35 (1999).

[4] R. Broda et al., Phys. Rev. Lett. 68, 1671 (1992).

[5] D. Bazzacco in: Proc. of the Int. Conf. on Nuclear Structure at High Angular Momentum, Ottawa, 1992, Vol.II, p.376, Report No. AECL10613

[6] C. Wheldon et al., Nucl. Phys. A699, 415 (2002).

[7] C. Wheldon et al., Nucl. Phys. A652, 103 (1999).

[8] D. Singh and D.A. Viggars, Nucl. Data Sheets 33, 275 (1981).

[9] C.Y. Wu et al., Nucl. Phys. A607, 178 (1996).

[10] C.Y. Wu et al., Phys. Rev. C64, 014307 (2001).

[11] R.A. Warner et al., Phys. Rev. Lett. 31, 835 (1973).

[12] B. Singh, Nucl. Data Sheets 99, 275 (2003).

[13] K. Jain et al., Nucl. Phys. A591, 61 (1995).

[14] C.M. Baglin, Nucl. Data Sheets 84, 717 (1988). 